\section{Evolutionary Optimization of Graphene-Metal Metasurfaces for Tunable Broadband Terahertz Absorption}

\author{
Elahe Sadat Torabi, Arya Fallahi and Alireza Yahaghi
}

are also studied: 1) the loss of the substrate, 2) structuring the substrate, and 3) single/double layer patches. The following section explains the design procedure in detail. Subsequently, section III outlines the results of the optimizations and finally, section IV concludes the letter.

\section{DESIGN}

Design process of a FSS absorber aims at the determination of substrate properties, patch configuration and array periodicity to achieve the optimal performance [21]. In this work, the frequency regime is fixed to the low THz. In addition, the height of the substrate and the periodicity of the array in both $\mathrm{x}\left(P_{x}\right)$ and $\mathrm{y}\left(P_{y}\right)$ directions are assumed to be 20 and $80 \mu \mathrm{m}$, respectively, and fixed during the whole optimizations.

Random initialized hill climbing algorithm (RHC) is chosen here to design the shape and material of the patch layer, due to the interesting properties outperforming many of the existing stochastic optimization methods for FSS design [21]. The details of this algorithm are presented in section II. B. The tunability of the absorber operation is obtained through introduction of graphene inclusions in the patch geometry. As proposed in [8], this tunablity can be achieved by controlling the conductance through an electrostatic bias field, which is applied normal to the surface. More details on this feature of graphene are presented in section II. A.

Besides a single layer FSS structure, double layer configuration is also investigated. In structures with single layer patch, a DC bias voltage is applied between the patch and the ground plane in order to provide the electrostatic bias field, while in structures with double layer patch, the second layer can function as a gate instead of the ground plane. As a result of smaller distance between the gates in the latter case in comparison to the former one, a considerable reduction in the required bias voltage is achieved [8]. Choosing a very thin substrate may similarly be helpful for this purpose. However, bringing the ground plane very close to the patch strongly affects the FSS performance [8].

Two distinct materials are selected for the substrate, silicon dioxide with a lossless relative permittivity $\varepsilon_{r}=4$ [9], and a lossy polymer with the relative permittivity $\varepsilon_{r}=3.5+0.2 j$ [17]. In addition to the usual homogeneous substrate, the effect of perforating the substrate, as a method for engineering its properties [4], is also examined. The optimum radius of the hole is determined by the optimization algorithm. Moreover, normal incidence is considered during all electromagnetic analyses.

\section{A. Graphene Model}

A graphene sheet is modeled by its tunable surface conductivity [14]. In the absence of spatial dispersion and magnetostatic bias field, one can model the conductivity by a scalar parameter [14]. Here, the spatial dispersion effect is negligible [22] and only the electrostatic bias is used to control the 


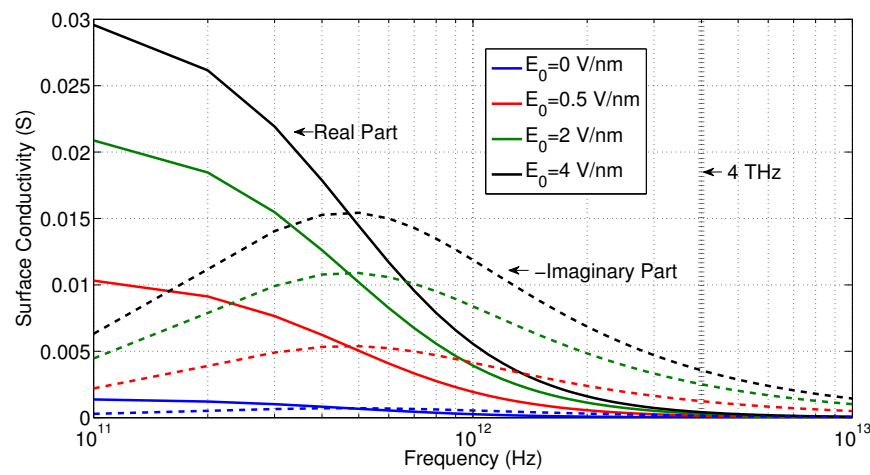

Fig. 1. The surface conductivity of graphene.The more bias voltage is applied, the more conductive the graphene surface will be. This dependency is stronger at low terahertz frequencies $(0.1-4 \mathrm{THz})$.

graphene conductivity. Therefore, the surface conductivity is determined by [14]:

$$
\begin{gathered}
\sigma\left(\omega, \mu\left(\mathbf{E}_{\mathbf{0}}\right), \Gamma, T\right)= \\
\frac{j e^{2}(\omega-j 2 \Gamma)}{\pi \hbar^{2}}\left\{\frac{1}{(\omega-j 2 \Gamma)^{2}}\right. \\
\int_{0}^{\infty} \varepsilon\left(\frac{\partial f_{d}(\varepsilon)}{\partial \varepsilon}-\frac{\partial f_{d}(-\varepsilon)}{\partial \varepsilon}\right) d \varepsilon \\
\left.-\int_{0}^{\infty} \frac{f_{d}(-\varepsilon)-f_{d}(\varepsilon)}{(\omega-j 2 \Gamma)^{2}-4\left(\frac{\varepsilon}{\hbar}\right)^{2}} d \varepsilon\right\} \\
f_{d}(\varepsilon)=\frac{1}{1+e^{\left(\varepsilon-\left|\mu_{c}\right|\right) / k_{B} T}}
\end{gathered}
$$

where $\omega$ is the frequency and $\Gamma$ is a phenomenological electron scattering rate which is assumed to be independent of the energy $\varepsilon$. In addition, $\mathrm{T},-e, \hbar=h / 2 \pi, f_{d}(\varepsilon)$, and $k_{B}$ stand for the temperature, the electron charge, the reduced Plank's constant, the Fermi-Dirac distribution (2), and the Boltzmann's constant, respectively. $\mu_{c}$ is also the chemical potential which is dependent on electrostatic bias field $\left(\mathbf{E}_{\mathbf{0}}\right)$. The phenomenological scattering rate can be written as $(1 / 2 \tau)$ [23], where $\tau$ represents the phenomenological scattering time equal to 0.34 ps [24], [25]. $\mathrm{T}$ is considered to be equal to the room temperature $(300 \mathrm{~K})$ and the regime of low field operation is assumed, which in turn justifies neglecting the nonlinear effects in graphene. The variation of surface conductivity in terms of frequency for different values of electrostatic bias field is shown in Fig. 1. It is observed that the graphene conductivity increases with the increase in the applied bias voltage. This property serves as a simple tuning mechanism based on electrostatic gating. Besides, the graphene nanoscale and planar structure is proper for miniaturization and integration especially for $\mathrm{THz}$ applications. To model graphene in HFSS [26], surface impedance $\left(Z_{s}=1 / \sigma\right)$ is assigned to the graphene sheet as a boundary condition [8], [23], [27].

\section{B. Optimization}

The surface of the unit cell is divided into $8 \times 8$ pixels (Fig. 2a). RHC algorithm uses an input bit string (binary code), where each bit determines the material assigned to the corresponding pixel. Presence and absence of a presumed

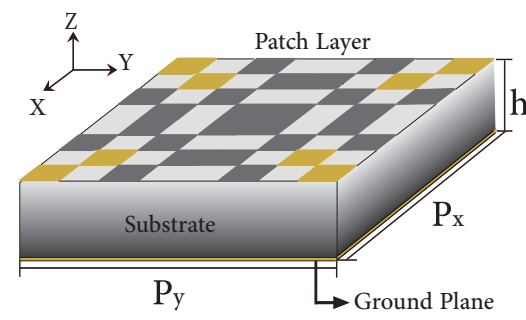

(a)

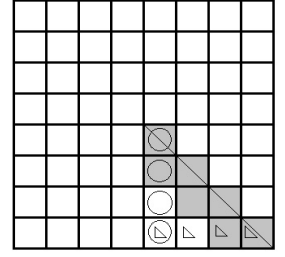

(b)
Fig. 2. a)The unit cell of the FSS absorber. Its surface is divided into $8 \times 8$ pixels. b)There are three locations (pixels marked by circles, the ones marked by triangles and the gray pixels) each of which must contain at least one bias element.

material at each pixel is represented by 1 and 0 , respectively. As mentioned above, for the sake of tunability, graphene is considered as a material in the patch layer. However, combination of graphene and other conventional conductors, like metals, increases the degrees of freedom in the design process. This in turn enables obtaining better optimum performances which are not feasible in a complete graphene based FSS. Therefore, four options are provided for a single pixel. They are gold, graphene with $\mu_{c}=0.775 \mathrm{eV}\left(E_{0}=4 \mathrm{~V} / \mathrm{nm}\right)$ which is tuned using the bias voltage, graphene with the same chemical potential which is fixed by chemical doping and air. A two-bit string is considered to determine the material type. More accurately, $\{00\},\{11\},\{10\}$ and $\{01\}$ represent gold, biased graphene, doped (unbiased) graphene and air, respectively. Finite conductivity as a boundary condition in HFSS is used to model the gold patches.

Mirror symmetries with respect to $x$ and $y$ axis as well as the diagonal symmetry is considered in the optimization (Fig. 2a). As a result, the electromagnetic response of the design will be independent of polarization under normal incidence [15]. Moreover, the whole patch configuration is determined once one eighth of the unit cell is known.

To apply bias voltage throughout the whole FSS surface, pixels to which biased graphene is assigned need to be electrically connected [8], [15]. The connection between these pixels may also be realized through gold inclusions. Consequently, a bias chain is obtained, which must be isolated from unbiased graphene pixels. One may think of shrinking the unbiased graphene pixels in order to prevent their connection to adjacent biased pixels. However, such a tiny margin hardly provides a safe isolation. Thus, the condition of 'no shared side with biased pixels' is imposed to unbiased graphene pixels.

To recognize the designs, in which correct connection between bias elements and isolation from unbiased pixels are foreseen, the following algorithm is followed:

- If there is no $\{11\}$ pixel, the design is correct.

- If the design contains $\{11\}$ pixels:

1) The presence of at least one $\{00\}$ or $\{11\}$ is essential at the specified locations in Fig. $2 \mathrm{~b}$ which are connecting gates to other pixels by symmetry and periodicity.

2) There must be at least one $\{11\}$ or $\{00\}$ pixel adjacent to a $\{11\}$ pixel, otherwise it is isolated. 
A depth-first search (DFS) algorithm is used in this step to register all connected pixels to the very first \{11\} pixel in the bit string and if any $\{11\}$ pixel remains separated from this chain after the search, the connection is lost.

3) Structures with $\{10\}$ pixel adjacent to the bias chain are rejected.

4) The $\{00\}$ pixels which met the first condition must be checked whether they are connected to the registered bias chain. If not, the connection is lost and the design is discarded.

Twelve optimizations with specific assumptions are carried out in this study. Two groups of designs need a detailed explanation:

1) Designs with double layer Patch: Both layers have the same pattern and two different gaps between the patch layers are examined, $1.68 \mu \mathrm{m}$ and $0.2 \mu \mathrm{m}$.

2) Designs with Structured Substrate: To structure the substrate, a hole is drilled at its center. The optimum radius of the hole is chosen by the optimization algorithm from discrete values between $4 \mu \mathrm{m}$ and $32 \mu \mathrm{m}$ with a step equal to $4 \mu \mathrm{m}$. Thus, three additional bits are needed to represent these eight radia in the bit strings. The height of the hole is set to $18 \mu \mathrm{m}$ to consider for a thin layer of the substrate which mechanically supports the patch layer.

\section{RESULTS}

The RHC algorithm stops when it reaches a maximum number of evaluations (1000 for the structures with homogeneous substrates and 1100 for the ones with perforated substrates). The optimum results are presented in Fig. 3.

The broadest bandwidth (1.95 THz) is achieved by the optimum design, in which the substrate is the perforated lossy polymer and the patch is single layer (Fig. $3 \mathrm{~m}$ ). The bit string of this design is $\{01011101110011011101111\}$. The last three bits stand for radius equal to $32 \mu \mathrm{m}$. Different absorption levels are obtained by applying various bias voltages to the biased graphene pixels of this design (Fig. 4).

\section{CONCLUSION}

Evolutionary optimization of graphene-metal metasurfaces is introduced as a promising approach to achieve designs meeting an application requirement. In this work, random hill climbing algorithm is used to find a graphene-based FSS design for tunable broadband $\mathrm{THz}$ absorption. Tunability of the designs is realized by including graphene patches which results in a simple tuning mechanism. Lossless and lossy substrates are examined as well as single and double layer patches. The broadest bandwidth of $1.95 \mathrm{THz}$ is obtained by the optimum design of a structure which has a perforated lossy substrate and a single layer patch.

\section{REFERENCES}

[1] K. Iwaszczuk, A. C. Strikwerda, K. Fan, X. Zhang, R. D. Averitt, and P. U. Jepsen, "Flexible metamaterial absorbers for stealth applications at terahertz frequencies," Optics express, vol. 20, no. 1, pp. 635-643, 2012.
[2] S. A. Kuznetsov, A. G. Paulish, A. V. Gelfand, P. A. Lazorskiy, and V. N. Fedorinin, "Matrix structure of metamaterial absorbers for multispectral terahertz imaging," Progress In Electromagnetics Research, vol. 122, pp. 93-103, 2012.

[3] N. Liu, M. Mesch, T. Weiss, M. Hentschel, and H. Giessen, "Infrared perfect absorber and its application as plasmonic sensor," Nano letters, vol. 10, no. 7, pp. 2342-2348, 2010.

[4] A. Fallahi, A. Yahaghi, H.-R. Benedickter, H. Abiri, M. Shahabadi, and C. Hafner, "Thin wideband radar absorbers," Antennas and Propagation, IEEE Transactions on, vol. 58, no. 12, pp. 4051-4058, 2010.

[5] F. Bayatpur and K. Sarabandi, "A tunable metamaterial frequencyselective surface with variable modes of operation," Microwave Theory and Techniques, IEEE Transactions on, vol. 57, no. 6, pp. 1433-1438, 2009.

[6] W. Hu, R. Dickie, R. Cahill, H. Gamble, Y. Ismail, V. Fusco, D. Linton, N. Grant, and S. Rea, "Liquid crystal tunable mm wave frequency selective surface," Microwave and Wireless Components Letters, IEEE, vol. 17, no. 9, pp. 667-669, 2007.

[7] M. H. Ucar, A. Sondas, and Y. E. Erdemli, "Switchable split-ring frequency selective surfaces," Progress In Electromagnetics Research $B$, vol. 6, pp. 65-79, 2008.

[8] A. Fallahi and J. Perruisseau-Carrier, "Design of tunable biperiodic graphene metasurfaces," Physical Review B, vol. 86, no. 19, p. 195408, 2012.

[9] R. Kitamura, L. Pilon, and M. Jonasz, "Optical constants of silica glass from extreme ultraviolet to far infrared at near room temperature," Applied optics, vol. 46, no. 33, pp. 8118-8133, 2007.

[10] T. Low and P. Avouris, "Graphene plasmonics for terahertz to midinfrared applications," ACS nano, vol. 8, no. 2, pp. 1086-1101, 2014.

[11] Y. V. Bludov, A. Ferreira, N. Peres, and M. Vasilevskiy, "A primer on surface plasmon-polaritons in graphene," International Journal of Modern Physics B, vol. 27, no. 10, p. 1341001, 2013.

[12] A. K. Geim and K. S. Novoselov, "The rise of graphene," Nature materials, vol. 6, no. 3, pp. 183-191, 2007.

[13] K. S. Novoselov, A. K. Geim, S. Morozov, D. Jiang, Y. Zhang, S. Dubonos, , I. Grigorieva, and A. Firsov, "Electric field effect in atomically thin carbon films," science, vol. 306, no. 5696, pp. 666-669, 2004.

[14] G. W. Hanson, "Dyadic green's functions for an anisotropic, nonlocal model of biased graphene," Antennas and Propagation, IEEE Transactions on, vol. 56, no. 3, pp. 747-757, 2008.

[15] A. Andryieuski and A. V. Lavrinenko, "Graphene metamaterials based tunable terahertz absorber: effective surface conductivity approach," Optics express, vol. 21, no. 7, pp. 9144-9155, 2013.

[16] B.-z. Xu, C.-q. Gu, Z. Li, and Z.-y. Niu, "A novel structure for tunable terahertz absorber based on graphene," Optics express, vol. 21, no. 20, pp. $23803-23811,2013$.

[17] Y. Zhang, Y. Feng, B. Zhu, J. Zhao, and T. Jiang, "Graphene based tunable metamaterial absorber and polarization modulation in terahertz frequency," Optics express, vol. 22, no. 19, pp. 22 743-22 752, 2014.

[18] M. Amin, M. Farhat, and H. Bağcı, "An ultra-broadband multilayered graphene absorber," Optics express, vol. 21, no. 24, pp. 29938-29948, 2013.

[19] H. Tao, C. Bingham, D. Pilon, K. Fan, A. Strikwerda, D. Shrekenhamer, W. Padilla, X. Zhang, and R. Averitt, "A dual band terahertz metamaterial absorber," Journal of physics D: Applied physics, vol. 43, no. 22, p. 225102,2010 .

[20] B. Xu, C. Gu, Z. Li, L. Liu, and Z. Niu, "A novel absorber with tunable bandwidth based on graphene," IEEE Antennas and Wireless Propagation Letters, vol. 13, pp. 822-825, 2014.

[21] A. Fallahi, M. Mishrikey, C. Hafner, and R. Vahldieck, "Efficient procedures for the optimization of frequency selective surfaces," Antennas and Propagation, IEEE Transactions on, vol. 56, no. 5, pp. 1340-1349, 2008.

[22] J. S. Gomez-Diaz, J. R. Mosig, and J. Perruisseau-Carrier, "Effect of spatial dispersion on surface waves propagating along graphene sheets," Antennas and Propagation, IEEE Transactions on, vol. 61, no. 7, pp. 3589-3596, 2013.

[23] J.-S. Gómez-Díaz and J. Perruisseau-Carrier, "Graphene-based plasmonic switches at near infrared frequencies," Optics express, vol. 21, no. 13, pp. 15 490-15 504, 2013.

[24] J. Y. Kim, C. Lee, S. Bae, K. S. Kim, B. H. Hong, and E. Choi, "Far-infrared study of substrate-effect on large scale graphene," Applied Physics Letters, vol. 98, no. 20, p. 201907, 2011.

[25] C. Lee, J. Y. Kim, S. Bae, K. S. Kim, B. H. Hong, and E. Choi, "Optical response of large scale single layer graphene," Applied Physics Letters, vol. 98, no. 7, p. 071905, 2011. 


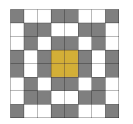

(a)

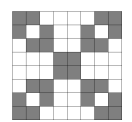

(b)

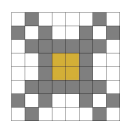

(c)

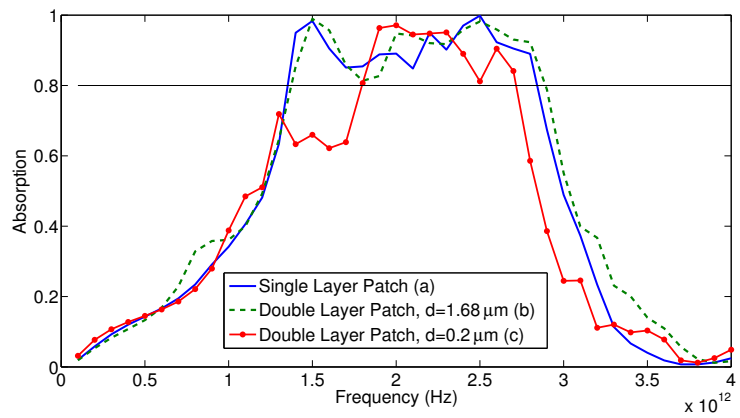

(d)

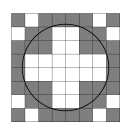

(i)

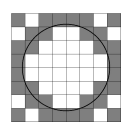

(j)

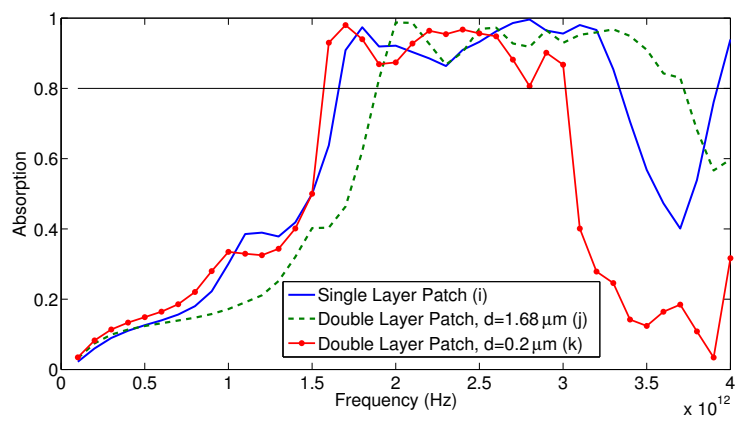

(1) (k)

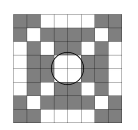

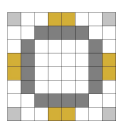

(e)

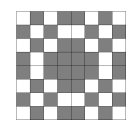

(f)

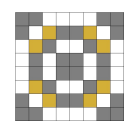

(g)

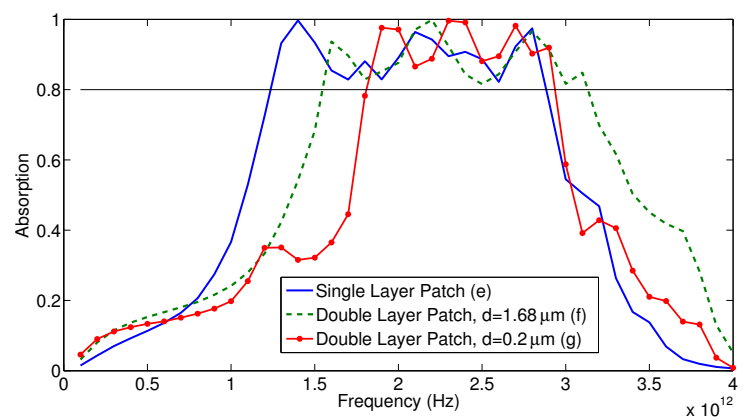

(h)

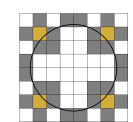

(m)

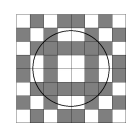

(n)

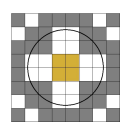

(o)

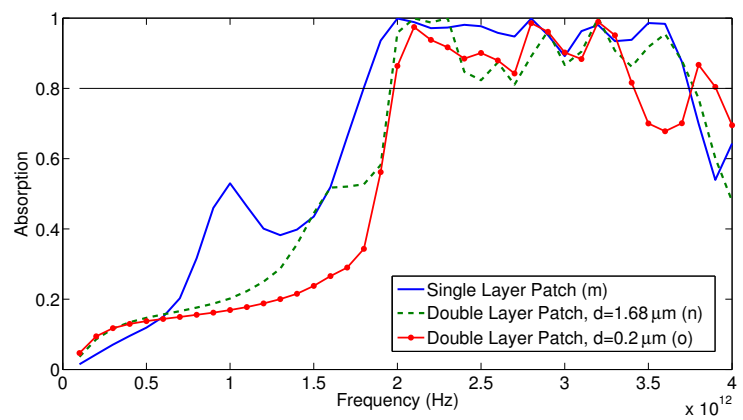

(p)

Fig. 3. Dark gray, bright gray and gold pixels represent biased graphene, unbiased graphene and gold, respectively. Schematics of the optimum designs of the structures with homogeneous silicon dioxide substrates: (a) single layer patch, double layer patch with (b) $\mathrm{d}=1.68 \mu m$ and (c) $\mathrm{d}=0.2 \mu m$. (d) The absorption versus frequency of the corresponding optimized structures (about 1.5, 1.55 and $0.95 \mathrm{THz}$ bandwidth for the schematics of (a), (b) and (d), respctively). Schematics of the optimum designs of the structures with homogeneous lossy polymer substrates: (e) single layer patch, double layer patch with (f) d=1.68 $\mu \mathrm{m}$ and (g) $\mathrm{d}=0.2 \mu \mathrm{m}$. (h) The absorption versus frequency of the corresponding optimized structures (about 1.65 , 1.6 and $1.1 \mathrm{THz}$ bandwidth for the schematics of (e), (f) and (g), respctively). Schematics of the optimum designs of the structures with perforated silicon dioxide substrates: (i) single layer patch, double layer patch with (j) $\mathrm{d}=1.68 \mu \mathrm{m}$ and $(\mathrm{k}) \mathrm{d}=0.2 \mu \mathrm{m}$. (1) The absorption versus frequency of the corresponding optimized structures (about $1.7,1.85$ and $1.5 \mathrm{THz}$ bandwidth for the schematics of (i), (j) and (k), respctively). Schematics of the optimum designs of the structures with perforated lossy polymer substrates: (m) single layer patch, double layer patch with (n) $d=1.68 \mu m$ and (o) $d=0.2 \mu m$. (p) The absorption versus frequency of the corresponding optimized structures (about 1.95, 1.85 and $1.45 \mathrm{THz}$ bandwidth for the schematics of (m), (n) and (o), respctively).

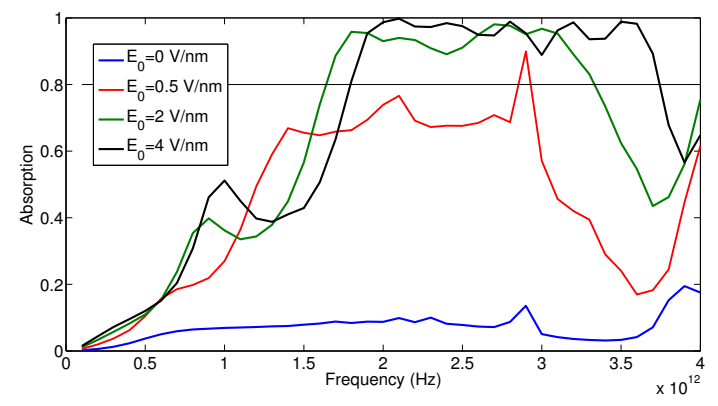

Fig. 4. Different absorption levels are obtained by applying various bias voltages to the the optimum design of the structure which has a perforated lossy polymer substrate and a single layer patch. This optimum design has the broadest bandwidth of $1.95 \mathrm{THz}$ when $\mu_{c}=0.775 \mathrm{eV}$.
[26] "High frequency structure simulator (hfss)," http://www.ansys.com/ Products/Electronics/ANSYS+HFSS.

[27] Y. R. Padooru, A. B. Yakovlev, C. S. Kaipa, G. W. Hanson, F. Medina, and F. Mesa, "Dual capacitive-inductive nature of periodic graphene patches: Transmission characteristics at low-terahertz frequencies," Physical Review B, vol. 87, no. 11, p. 115401, 2013. 